\title{
Research for Classification Method of Battery Based on State of Health
}

\author{
Yu Zhilong and Li Ran \\ School of Automation, Harbin University of Science and Technology, Harbin, \\ China \\ E-mail: zlyu@hrbust.edu.cn
}

\begin{abstract}
In order to solve the problems that lithium-ion power battery cannot reflect state of health(SOH) in sorting process, the parameters which can reflect the battery $\mathrm{SOH}$, such as capacity, AC resistance and self-discharge current, were used as the input vector of battery sorting model, fuzzy c-means clustering analysis and support vector machine based on cross validation algorithm to the battery for classification and recognition were used, and lithium power battery sorting model was established and the same batch of power battery were separating tested and according to the experimental results, batteries was divided into groups. The test results showed that: battery electrochemistry was having a good consistency. The variation of capacitance was less than 5\% while there was 1500 cycle life.
\end{abstract}

Keywords: power battery; state of health; fuzzy c-means clustering analysis; SVM

\section{Introduction}

There are many parameters reflecting the lithium power battery capacity performance [1] and health status, including physical properties, electrochemical properties. After the end of the battery package production and formation, we need combination classification of the battery characteristics, to meet the needs of grouping of battery. Electrochemical consistency detection accuracy directly affect battery performance [2] after grouping. The parameter of battery characteristics mainly includes: the quality of the battery, the open circuit voltage, discharge plateau voltage, current, depth of discharge, the rated capacity, AC resistance, self-discharge rate and $\mathrm{SOC} \mathrm{SOH}$. The SOC, $\mathrm{SOH}$ is directly reflecting the power of battery and the degree of deterioration, but in practical application process there is not a particularly good way to measure, and parameters which can be measured can be the basis for the classification of industrial field. Due to the wide use of power battery, the core technology of the management system which can improve monomer battery electrochemical consistency and improve the efficiency of the battery group demands an urgent improvement. Thus, electrochemical separation technology considering battery group measured volume has become a hot topic in the research of battery technology. Conventional battery classification methods are: Static volumetric method, voltage assembly method, charge and discharge efficiency method, internal matching method, internal resistance and capacity sorting, cyclic data test method. Multicriteria sorting method [3-13]. Paolo Raspaand the others [3] in order to improve the cell uniformity, used self-organizing map neural network method, to develop a method for the homologous cell selection and classification. Zhihua Duo, Gechen Li [4], put forward a strategy of rapid cell classification, using fuzzy decision making comparative analysis of battery charge and discharge curves and discharge capacity, and completed a sorting of battery consistency .

Due to the difference in the battery production process in control of electrochemical characteristics and packaging technology, the electrochemical properties of the battery of 
the same formula and the same batch will differ [10], and the electrochemical difference does not have a very accurate standard to measure the electrochemical characteristics of the monomer battery belongs to which category, so in the process of grouping, cell sorting is only according to certain rules of sorting, as far as possible to reflect the electrochemical cell consistency[14-15].

\section{Battery Classification Model}

Due to the battery in the classification process does not have a quantitative classification standard, each classification is as far as possible to improve the consistency of the electrochemical cell in the same category. Therefore, the selection of parameters reflecting the electrochemical characteristic and categories of the correct division of the battery is the key to battery classification technology. So the main idea of classification method in the essay is: use data mining techniques to obtain cell classification data, and use fuzzy c-means clustering algorithm to determine the cluster centers of the cell samples, and use characteristics of the sample to determine cell classification numbers and use SVM calculation method to the category formed by the sample for discriminative training and classification [16-17].

\subsection{Battery Parameter Selection}

Considering the work characteristics of electric core in the batteries will reflect the battery deterioration of the AC resistance, self-discharge current and actual capacity, SOC and measurable quantities in battery operation process: use the open circuit voltage, discharge plateau voltage, voltage change rate in the process of discharging as battery electrochemical separation sample data of battery sorting, and obtain the sample set for

$\boldsymbol{x}_{i}=\left[V_{\mathrm{o}}, V_{\mathrm{dis}}, S O C, \Delta V, R, m, I_{\text {sdis }}, P\right]$

Among them, $V_{\mathrm{o}}$ is open circuit voltage, $V_{\text {dis }}$ is discharge plateau voltage, $\Delta V$ is voltage change rat; $R$ is AC resistance, $m$ is the quality of battery, $I_{\text {sdis }}$ is selfdischarge current, $P$ is actual discharge capacity of battery.

Using the eight measurable quantities adopted by formula (1) as the sample data of battery classification can fully reflects the battery's electrochemical and physical properties, and the AC resistance of battery, self-discharge current reflects the battery's SOH, discharge capacity and the open circuit voltage, and voltage of discharge platform reflects the life of the battery, and SOC and voltage changing rate in the course of the discharging is a reflection of the dynamic characteristics of the battery but cycle, and battery quality reflects the physical quantity of active substances of the cell. So cell sample classification set formed by the actual measurement parameters of the battery can fully reflect characteristics of the battery.

\subsection{Establishment of FCM-SVM classification model}

The specific steps to be established based on the FCM-SVM battery classification model are as follows:

Step 1, Use the fuzzy $\mathrm{C}$ mean clustering algorithm to classify the all learning samples of battery, get the clustering center $m_{i}, i=1,2, \ldots, c$,

Step 2, Calculate the Euclidean distance between cluster centers, i.e.,

$$
d_{i, j}=\left\|m_{i}-m_{j}\right\|, i, j=1,2, \ldots, c, i \neq j
$$


Step 3, Calculate distance between each clustering center and other clustering centers, and order and number them according to the distance, get distance between $d_{i, j}$ with other clustering centers.

$$
p_{i}^{1} \leq p_{i}^{2} \leq \ldots \leq p_{i}^{c-1}
$$

Step 4, Compare the minimum distance of different clustering centers, then have the cluster centers ranked according to the distance. The clustering centers are ranked according to the distance from large to small sort, and we get a new clustering center label for $M_{1}, M_{2}, \ldots, M_{c}$.

Step 5, First set the sample sequence set corresponding to $M_{1}$ to be the positive class, and set sample sequence sets corresponding to other clustering centers to be the negative class, then structure support vector machine $S V M_{1}$, and set the sample sequence set corresponding to $M_{1}$ to be the positive class, $M_{3}, M_{4}, \ldots, M_{c}$ to be the negative class, and structure support vector machine $S V M_{2}$, etc., finally get $c-1$ support vector machine classifier.

In step 5 , we need to build $c-1$ support vector machine (SVM) classifier and a single binary support vector machine using C-SVC model to train and specific forms are as follows:

Step 1, Set a known training set:

$$
T=\left\{\left(\boldsymbol{x}_{i}, y_{i}\right)\right\} \in(X \times Y)^{l}
$$

Among them, $\boldsymbol{x}_{i}$ is cell sample collection, and $\boldsymbol{x}_{i} \in X=\boldsymbol{R}^{8}, \quad y_{i} \in Y=\{1,-1\}$, $i=1,2, \ldots, l$

After the training samples and test samples were normalized, have the samples normalize into the range of $[0,1]$.

Step 2, The SVM kernel function is selected as the radial basis function.

$$
K\left(\boldsymbol{x}, \boldsymbol{x}_{i}\right)=\exp \left(-\gamma\left\|\boldsymbol{x}-\boldsymbol{x}_{i}\right\|^{2}\right), \gamma>0
$$

Step 3, According to the cross validation method to choose the appropriate penalty factor, radial basis function parameter. Train support vector machine classifier, get the model and test it.

Use fuzzy $\mathrm{C}$ means clustering analysis and support vector machine algorithm based on cross validation are used to classify the battery. The specific model is shown in Figure 1. 


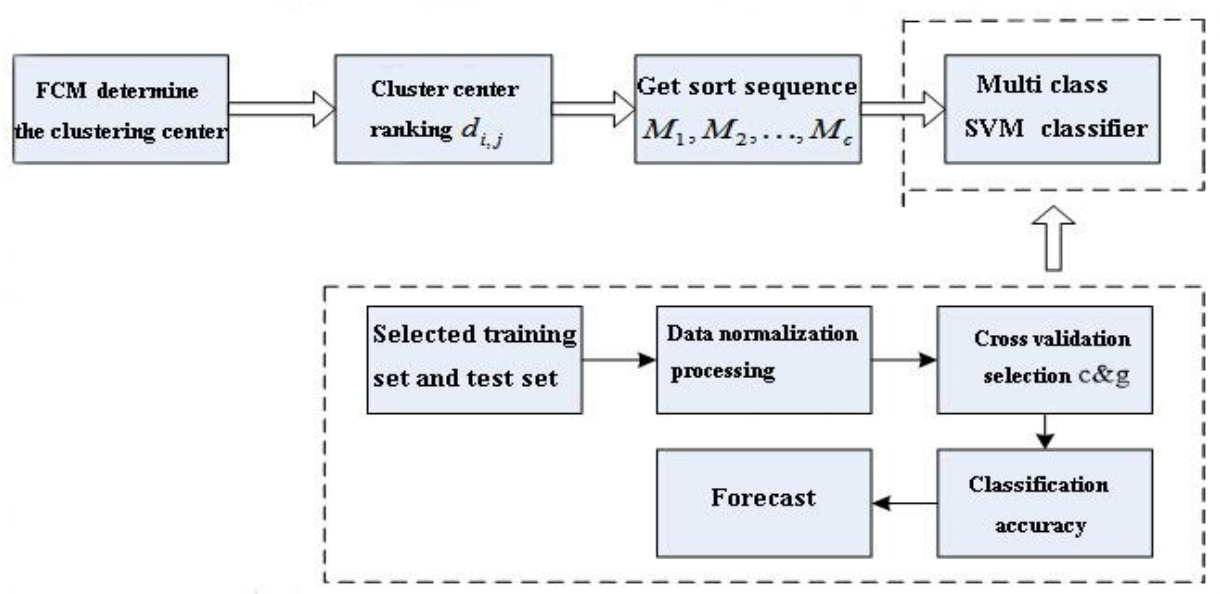

Figure 1. SVM Algorithm based on FCM

\section{Verification of Classification Experiment}

\subsection{Data collection}

Experiments are using Arbin battery charging and discharging equipment and the experimental object is 120 batches of IFR18650P lithium iron phosphate monomer battery, and its rated capacity is of $1100 \mathrm{mAh}$.

Experimental way: under the room temperature of $20^{\circ} \mathrm{C}$, using standard current $1 \mathrm{C}$ of battery for charging and discharging experiments, in small current of $1 \mathrm{c}$ we think that battery charge and discharge efficiency is $100 \%$. The charge and discharge current should be kept constant, and the ampere hour method is used to calculate SOC value; after electric core charging is completed and SOC is $100 \%$, let the battery stew for 30 minutes, and record the battery terminal voltage in static state and measure the $\mathrm{AC}$ resistance, quality of electric core; in the discharge, record voltage of electric core discharging platform, voltage changing rate and discharging capacity of the battery. In different SOC of battery, measure self-discharge flow of electric core randomly, and finally get the 220groups of measurement data, as shown in Table 1

Table 1. Sample Data of Battery

\begin{tabular}{|c|c|c|c|c|c|c|c|}
\hline \multirow[b]{2}{*}{ Samples } & \multicolumn{7}{|c|}{ Parameters } \\
\hline & $\mathrm{OCV} / \mathrm{V}$ & $\mathrm{DPV} / \mathrm{V}$ & SOC & $\begin{array}{c}\mathrm{AC} \\
\text { Resistance } \\
/ \mathrm{m} \Omega\end{array}$ & Quality/mg & $\begin{array}{c}\text { Self- } \\
\text { discharge } \\
\text { current/ } \mu \mathrm{A}\end{array}$ & $\begin{array}{l}\text { Power } \\
/ \mathrm{mAh}\end{array}$ \\
\hline 1 & 3333.5 & 3221.6 & $94.25 \%$ & 64.6 & 39.9 & 23.1 & 1094.0 \\
\hline 2 & 3323.2 & 3223.6 & $92.09 \%$ & 66.1 & 40.0 & 27.3 & 1077.4 \\
\hline 3 & 3333.7 & 3222.3 & $92.30 \%$ & 63.5 & 39.7 & 30.1 & 1083.5 \\
\hline 4 & 3220.0 & 3122.3 & $94.65 \%$ & 56.8 & 39.9 & 45.0 & 1157.3 \\
\hline 5 & 3221.8 & 3112.5 & $97.34 \%$ & 57.4 & 40.0 & 46.2 & 1156.2 \\
\hline
\end{tabular}

\subsection{Model Training and Simulation}

Use 220 groups of data as the training and testing data for battery classification 
model, including 120 groups as the training data, another 100 groups for the test data. Have data clustering analysis with FCM algorithm, and the clustering result curve as shown in Figure 2.

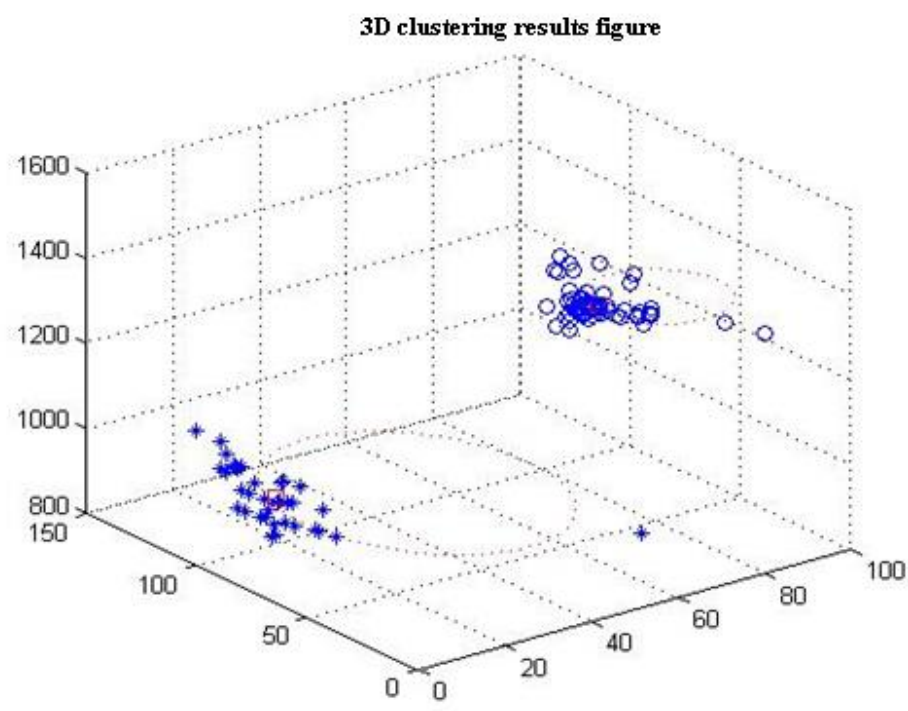

Figure 2. Results of Cluster Analysis

According to the result of the clustering analysis, using the 120 sets of data for iteration, we got the most reasonable classification 2classe, as shown in Figure 2. Through clustering analysis for measured data we obtained 2 class, and eliminated the differences, and with usage of SVM classifier we could classify the sample cell data.

In the training using LIBSVM Matlab toolbox to train two classifiers, the training samples adopted the 120 groups of known data in the measuring samples, and used the remaining 100 sets of data for cross validation. Validation results are shown in Figure 3.

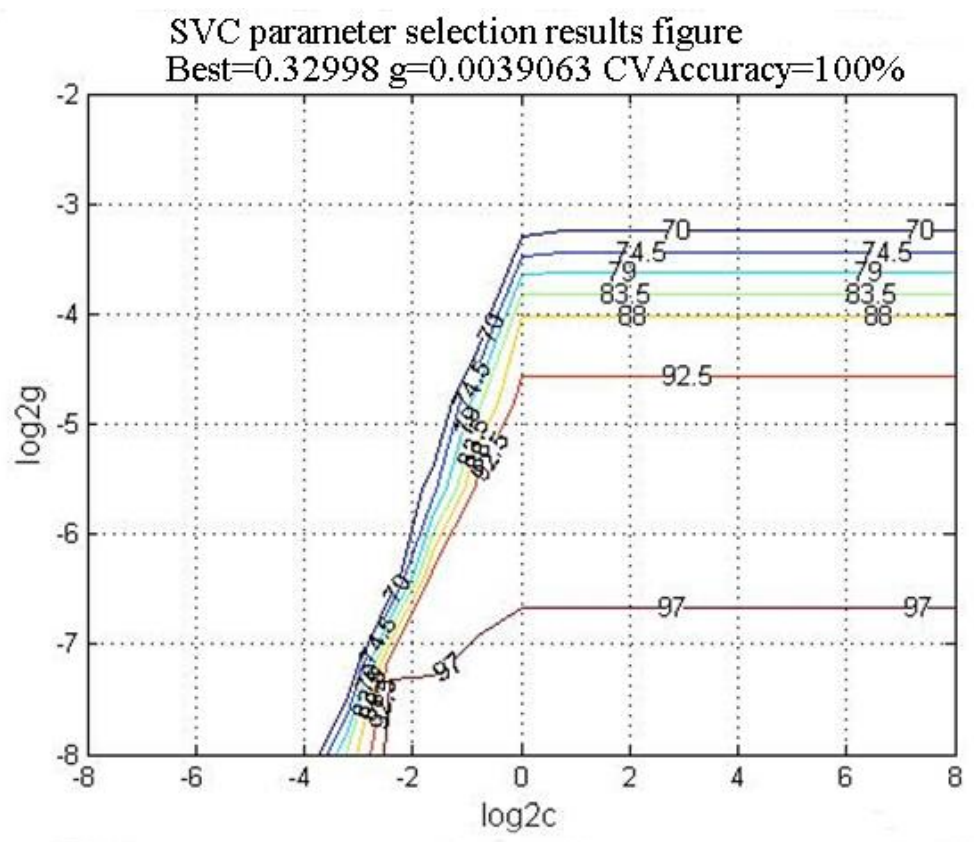

Figure 3. Contour Map of Parameter Selection 
As is shown in Figure 3, by cross validation, the optimum parameters $g=0.0039063 c=0.32988$ was selected. Using optimal parameters to train the classification model, we got the $100 \%$ classification accuracy rate.

\section{Experimental Verification}

To verify the correctness of battery sorting results, we had a battery sorting of the same batch in accordance with the model, and took 10 in the same category for battery cycle life experiment. Experimental scheme is: under the temperature of $20^{\circ} \mathrm{C}$, have $1 \mathrm{C}$ charge and discharge experiments on the battery respectively, with charge cutoff voltage of $3.2 \mathrm{~V}$, discharge cut-off voltage of $2.0 \mathrm{~V}$, and record the discharge capacity of the battery each interval of 50 cycles. The result is shown in Figure 4.

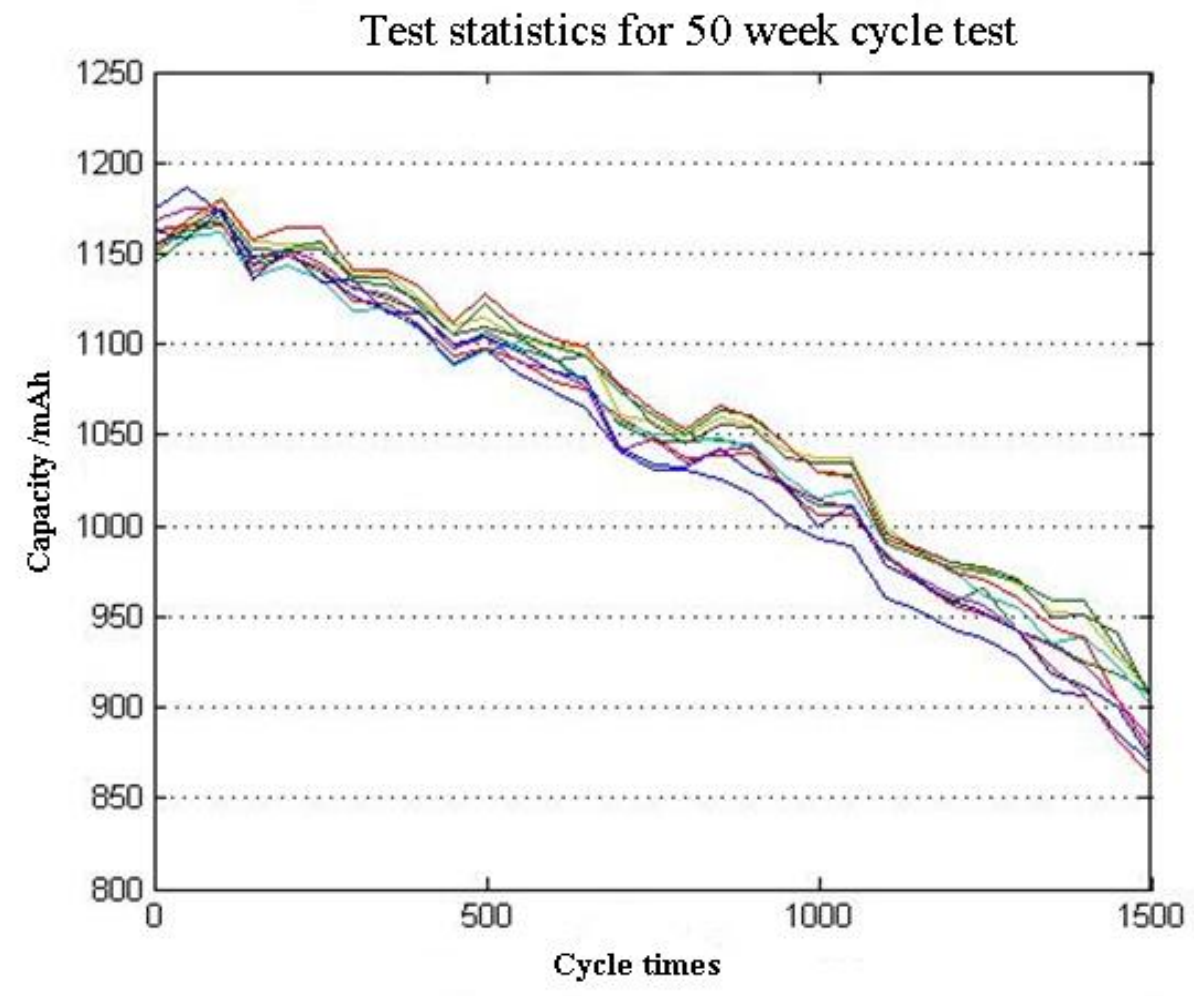

Figure 4. Discharge Capacity of Battery in Cycle Testing

As is shown in chart 5, battery classification algorithm can better reflect the battery electrochemical consistency In cycle life experiment of 10 batteries, the battery capacity can maintain a good attenuation trend after 1500 cell cycles, and with the relative dispersion of battery capacity in a single measurement, capacity difference maximum less than 5\% can indicate that cell electrochemistry is in good consistency. Battery sorting method based on FCM-SVM can achieve satisfactory effect.

\section{Conclusion}

This essay adopts capacity, internal resistance and self-discharge current which can reflect the electrochemical properties of lithium ion power battery as the input parameters of the battery model to construct the battery classification model. Experimental results shows that using the battery sorting results which adopted this essay's classification algorithm can better take into account the battery health effects but the main problems is battery parameter measurement in the classification algorithm will take a long time and is not suitable for large quantities of industrial field application. 


\section{Acknowledgement}

This paper is partially supported by Technological Innovation Foundation for Leaders of Disciplines in Science of Harbin (2014RFXXJ032).

\section{References}

[1] RAMADASS P, HARAN Bala and WHITE Ralph, "Mathematical modeling of the capacity fade of Liion cells [J]", Journal of Power Sources, vol. 123, (2003), pp. 230-240.

[2] L. Ran, W. Junfeng and W. Haiying, "Research on consistency classification of battery based on multi point spectrum method", Power technology, vol. 10, (2010), pp. 19-22.

[3] P. Raspa, L. Frinconi and A. Mancini, "Selection of Lithium Cells for EV Battery Pack Using SelfOrganizing Maps", J Automotive Safety and Energy, vol. 2, no. 2, (2011), pp. 157-164.

[4] D. Zhihua and L. Gechen, "An algorithm for the identification of the power of waveform recognition", Power technology, vol. 22, no. 4, (1998), pp. 174-178.

[5] W. Yao, "Battery consistency matching technology for electric vehicles", Electrical industry, vol. 6, (2002), pp. 15-17.

[6] C. Shaoan, Z. Jianqing and L. Hong, "Electrochemical impedance spectroscopy (MH-Ni) analysis of cell cycle decline", Power supply technology, vol. 23, (1999), (S1) pp. 62-64.

[7] R. Li, J. Wu and H. Wang, "Research on battery consistency classification based on multi point spectrum method", Power supply technology, vol. 10, (2010), pp. 19-22.

[8] Y. Linru, Z. Jinge and W. Yan, "MH-Ni battery classification method based on data fusion theory", Power supply technology, vol. 3, (2000), pp. 25-28.

[9] K. Dae-Keun and S. Heon-Cheol, "Investigation on cell impedance for high-power lithium-ion batteries [J]", J Solid State Electrochem, vol. 11, (2007), pp. 1405-1410.

[10] G. Weijun, S. Zechang and W. Xuezhe, "Two Methods Studying Internal Resistance for Lithium-ion Battery Life Estimation Used in EV [J]", J Automotive Safety and Energy, vol. 2, no. 2, (2011), pp. 145149.

[11] H. Wang, L. He and J. Sun, "Study on correlation with SOH and EIS model of Li-ion battery $[\mathrm{C}] / /$ Strategic Technology (IFOST)", 2011 6th International Forum on. IEEE, vol. 1, (2011), pp. 261264.

[12] W. Haiying, L. Yang and F. Hang, "Estimation of state of charge of batteries for electric vehicles [J]", International Journal of Control and Automation, vol. 6, no. 2, (2013), pp. 185-194

[13] Z. Chen, "Design and SOC estimation of lithium ion battery monitoring and management system for electric vehicle [D]", Nanjing University of Science and Technology, (2013), pp. 19-30.

[14] W. Haiying, X. Chao and F. Qi, "Lithium Battery SOC Estimation Method Study Based on Principal Component Analysis[J]", International Journal of Control and Automation, vol. 8, no. 7, (2015), pp. 185-192.

[15] X. Hui, "Study on SOH estimation method of power Li ion battery [D]", Jilin University, (2013).

[16] Y. Yang, "Study on the method of fuzzy C mean clustering in fuzzy clustering analysis [D]", Shenyang: Shenyang University of Technology, (2009).

[17] Y. Haijun, "Research on fuzzy clustering analysis technology and its application [D]", Hefei: He Fei University of Technology, (2006). 
International Journal of Security and Its Applications

Vol. 10, No. 6 (2016) 